\title{
Abundances in planetary nebulae: NGC 2792^
}

\author{
S. R. Pottasch ${ }^{1}$, R. Surendiranath ${ }^{2}$, J. Bernard-Salas ${ }^{3}$, and T. L. Roellig ${ }^{4}$ \\ 1 Kapteyn Astronomical Institute, P.O. Box 800, NL 9700 AV Groningen, The Netherlands \\ e-mail: pottasch@astro.rug.nl \\ 2 Indian Institute of Astrophysics, Koramangala II Block, Bangalore-560034, India \\ 3 Center for Radiophysics and Space Research, Cornell University, Ithaca, NY 14853, USA \\ 4 NASA Ames Research Center, MS 245-6, Moffett Field, CA 94035-1000, USA
}

Received 16 March 2009 / Accepted 15 May 2009

\section{ABSTRACT}

\begin{abstract}
The mid-infrared spectrum of the rather circular planetary nebula NGC 2792 taken with the Spitzer Space Telescope is presented. This spectrum is combined with the ultraviolet $I U E$ spectrum and with the spectrum in the visual wavelength region to obtain a complete, extinction corrected, spectrum. The chemical composition of the nebula is then calculated in two ways. First by directly calculating and adding individual ion abundances, and secondly by building a model nebula that attempts to reproduce the observed spectrum. Because it is now possible to include the nebular temperature gradient, the chemical composition is more accurate than has been given earlier in the literature. Discussion of both the central star and the evolution of the star-nebula is then given.
\end{abstract}

Key words. ISM: abundances - planetary nebulae: individual: NGC 2792 - infrared: ISM - ISM: lines and bands

\section{Introduction}

NGC 2792 (PK 265.7+04.1) is a rather average planetary nebula. An image in visible light is shown as Fig. 1. The nebula is usually classified as elliptical although it is almost circular in shape. In addition it contains several structural details. A halo is present as has been measured by Corradi et al. (2003). These authors measure a radius of $5.5^{\prime \prime}$ for the bright inner part of the nebula, which is surrounded by a fainter nebula which decreases in brightness to about a radius of $10^{\prime \prime}$. The halo continues at a very low surface brightness (less than $10^{-3}$ of the brightness of the main nebula) until a radius of about $37^{\prime \prime}$.

The distance of the nebula is very uncertain. Statistical distances are between $1.4 \mathrm{kpc}$ and $3 \mathrm{kpc}$. By equating the $\langle\mathrm{rms}\rangle$ density with the forbidden line density, a value of $d=1.5 \mathrm{kpc}$ is found and used when necessary throughout this paper. This distance is close to the value of $1.9 \mathrm{kpc}$ found by the extinction method by Gathier et al. (1986). Adopting this value, the nebula is thus about $100 \mathrm{pc}$ above the galactic plane.

The purpose of this paper is to obtain accurate abundances for this nebula. This is achieved in two ways. First by including the mid-infrared spectrum taken with the IRS spectrograph of the Spitzer Space Telescope (Werner et al. 2004). The reasons for this have been discussed in earlier papers (e.g. see Pottasch \& Beintema 1999; Pottasch et al. 2000, 2001; Bernard Salas et al. 2001), and can be summarized as follows: 1) the intensity of the infrared lines is not very sensitive to the electron temperature nor to possible extinction effects; 2) use of the infrared line intensities enable a more accurate determination of the electron temperature for use with the visual and ultraviolet lines; 3) the number of observed ionization stages is doubled.

* Based on observations with the Spitzer Space Telescope, which is operated by the Jet Propulsion Laboratory, California Institute of Technology.

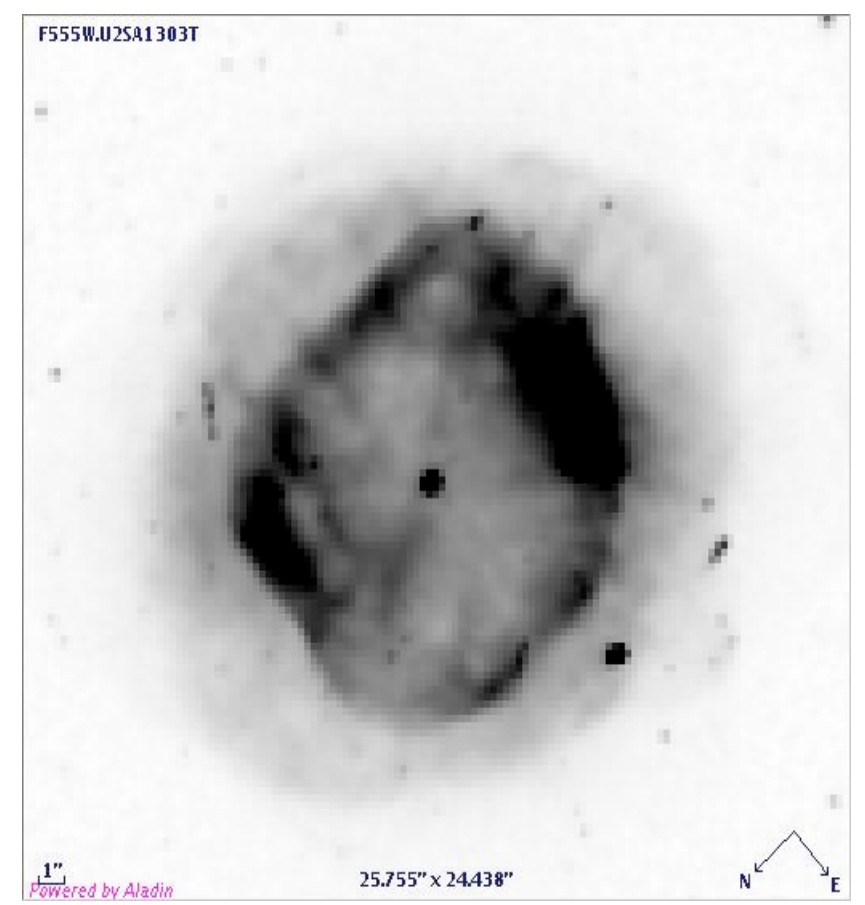

Fig. 1. HST image of NGC 2792; Credits: Howard Bond; HST archived exposures catalog (STSCI, 2007).

The second method of improving the abundances is by using a nebular model to determine them. This has several advantages. First it provides a physical basis for the electron temperature determination. Secondly it permits abundance determination for elements which are observed in only one, or a limited number of ionic stages. This is true of $\mathrm{Mg}, \mathrm{Si}$, and $\mathrm{Cl}$, which would be less reliably determined without a model. A further advantage of 
modeling is that it provides information on the central star and other properties of the nebula.

A disadvantage of modeling is that there are more unknowns than observations and some assumptions must be made; for example, concerning the geometry, we will assume that the nebula is spherical and that no clumping exists. The observed circular form and smooth emission make these assumptions reasonable. Furthermore we are limited in the form of the radiation field of the exciting star. Other assumptions are discussed in Sect. 4.1.

Abundance determination for this nebula has been made earlier. The most extensive are the works of Milingo et al. (2002a,b; see also Henry et al. 2005), and Kingsburgh \& Barlow (1994). A more limited number of elements have been studied by TorresPeimbert \& Peimbert (1977) and by de Freitas Pacheco et al. (1992). These authors give differing and incomplete abundance values; our purpose is to bring order in this situation.

This paper is structured as follows. First the spectrum of NGC 2792 is discussed (in Sect. 2). Section 3 discusses the simple approach to determining the chemical composition and presents the resultant abundances. In Sect. 4. the model assumptions are detailed and the results are presented. The results of the model are discussed in detail in Sect. 5. The abundances determined are compared with those in the literature in Sect. 6, which also includes a discussion of the errors. In Sect. 7 a discussion of the central star and the evolutionary state of the nebula is given. Finally, our conclusions are given in Sect. 8.

\section{The spectrum of NGC 2792}

\subsection{The infrared spectrum}

Observations of NGC 2792 were made using the Infrared Spectrograph (IRS, Houck et al. 2004) on board the Spitzer Space Telescope with AOR keys of 4111104 (on target) and 4111360 (background). The reduction started from the droop images which are equivalent to the most commonly used Basic Calibrated Data $(b c d)$ images but lack stray-cross removal and flat-field. The data were processed using the $\mathrm{s} 15.3$ version of the pipeline and using a script version of Smart (Higdon et al. 2004). The tool irsclean was used to remove rogue pixels. The different cycles for a given module were combined to enhance the $\mathrm{S} / \mathrm{N}$. At this point the background images were subtracted to remove the sky contribution. We note that since we are interested in line fluxes the removal of background is irrelevant for our analysis except for aiding in the removal of any rogue pixel that may have been left out by the irsclean tool (i.e. those with low flux). Then the resulting high-resolution modules (HR) were extracted using full aperture measurements.

The IRS high resolution spectra have a spectral resolution of about 600 , which is a factor of between 2 and 5 less than the resolution of the ISO SWS spectra. The mid-infrared measurements are made with several different diaphragm sizes. Because the diaphragms are smaller than the size of the nebulae and are all of differing size, we first discuss how the different spectra are placed on a common scale.

Two of the three diaphragms used have high resolution: the short high module (SH) measures from $9.9 \mu \mathrm{m}$ to $19.6 \mu \mathrm{m}$ and the long high module (LH) from $18.7 \mu \mathrm{m}$ to $37.2 \mu \mathrm{m}$. The $\mathrm{SH}$ has a diaphragm size of $4.7^{\prime \prime} \times 11.3^{\prime \prime}$, while the LH is $11.1^{\prime \prime}$ $\times 22.3^{\prime \prime}$. If the nebulae are uniformly illuminating then the ratio of the intensities would simply be the ratio of the areas measured by the two diaphragms. Since this is not so, we may use the ratio of the continuum intensity in the region of wavelength overlap at $19 \mu \mathrm{m}$. These continua are equal when the $\mathrm{SH}$

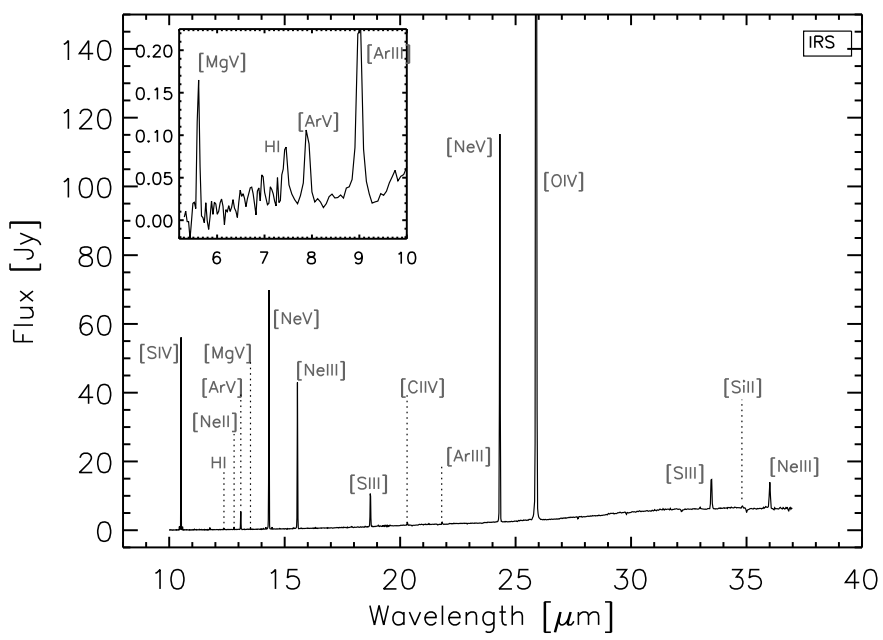

Fig. 2. The IRS spectrum of NGC/,2792. The SH intensity (from $9.9 \mu \mathrm{m}$ to $19.6 \mu \mathrm{m}$ ) has been scaled by 2.65 to match $\mathrm{LH}$ (from $18.7 \mu \mathrm{m}$ to $37.2 \mu \mathrm{m}$ ). The SL spectrum between 5 and $10 \mu \mathrm{m}$ is shown in the inset and has not been scaled.

intensities are increased by a factor of 2.65. The third diaphragm is a long slit which is $4^{\prime \prime}$ wide and extends over the entire nebula. This SL module measures in low resolution and measures between $5.5 \mu \mathrm{m}$ and $14 \mu \mathrm{m}$. These spectra are normalized by making the lines in common between the SL and SH modules to agree. Especially important is the agreement of the [S IV] line at $10.51 \mu \mathrm{m}$. The spectrum is shown in Fig. 2.

The IRS measurement of NGC 2792 was centered at $\operatorname{RA}(2000) 09^{\mathrm{h}} 12^{\mathrm{m}} 26.63^{\mathrm{s}}$ and $\operatorname{Dec}(2000)-42^{\circ} 25^{\prime} 40.1^{\prime \prime}$. This is almost exactly the same as the value measured by Kerber et al. (2003) of $\operatorname{RA}(2000) 09^{\mathrm{h}} 12^{\mathrm{m}} 26.60^{\mathrm{s}}$ and $\operatorname{Dec}(2000)$ $-42^{\circ} 25^{\prime} 39.9^{\prime \prime}$, which is presumably the coordinate of the central star. Thus the IRS measurement was well centered on the nebula. The fluxes were measured using the Gaussian line-fitting routine. The measured emission line intensities are given in Table 1, after correcting the $\mathrm{SH}$ measurements by the factor 2.65 and the SL measurements by a factor of 5.5, in the column labeled "intensity". The $\mathrm{H} \beta$ flux found from the infrared hydrogen lines (especially the lines at $12.37 \mu \mathrm{m}$ ) using the theoretical ratios of Hummer \& Storey (1987), is $2.92 \times 10^{-11} \mathrm{erg} \mathrm{cm}^{-2} \mathrm{~s}^{-1}$, which is about $95 \%$ of the total $\mathrm{H} \beta$ intensity. This is reasonable since the well-centered LH diaphragm coves much of the nebula.

\subsection{The visual spectrum}

The visual spectrum has been measured by four authors. We list here the results. The line intensities listed have been corrected by each author for a value of extinction determined by them to obtain a theoretically correct Balmer decrement. The results are listed in Table 2, where the last column lists the average value which we have used. No attempt has been made to use a common extinction correction because then the Balmer decrement will be incorrect. The value of extinction $\mathrm{C}$ which the individual authors found is listed at the bottom of the table. None of the spectra measure the weaker lines very well. The errors may be judged by the agreement (or disagreement) of the various measures and appear to be within $20 \%$ for the stronger lines and probably much worse for the weaker lines. 
Table 1. IRS spectrum of NGC 2792.

\begin{tabular}{lccc}
\hline \hline Identification & $\lambda(\mu \mathrm{m})$ & Intensity $^{\dagger}$ & $I / \mathrm{H} \beta$ \\
\hline$[\mathrm{Mg} \mathrm{V}]$ & 5.606 & $332 \pm 21$ & 11.5 \\
$\mathrm{H} \mathrm{I} \mathrm{(6-5)}$ & 7.478 & $89 \pm 14$ & 3.05 \\
{$[\mathrm{Ar} \mathrm{V}]$} & 7.904 & $171 \pm 8$ & 5.9 \\
{$[\mathrm{Ar} \mathrm{III}]$} & 8.997 & $231 \pm 7$ & 7.9 \\
{$[\mathrm{~S} \mathrm{IV}]$} & 10.510 & $3140 \pm 50$ & 108 \\
$\mathrm{H} \mathrm{I}(9-7)$ & 11.304 & $9.62: \pm 1.2$ & 0.33 \\
{$[\mathrm{Cl}$ IV] } & 11.763 & $26.1 \pm 4.0$ & 0.894 \\
$\mathrm{H} \mathrm{I} \mathrm{(7-6+11-8)}$ & 12.371 & $25.6: \pm 3.8$ & 0.877 \\
{$[\mathrm{Ne} \mathrm{II}]$} & 12.811 & $28.8 \pm 1.0$ & 0.986 \\
{$[\mathrm{Ar} \mathrm{V}]$} & 13.099 & $206 \pm 5$ & 7.07 \\
{$[\mathrm{Mg} \mathrm{V}]$} & 13.518 & $19.4: \pm 1.2$ & $0.664:$ \\
{$[\mathrm{Ne} \mathrm{V}]$} & 14.320 & $2700 \pm 90$ & 92.6 \\
{$[\mathrm{Fe} \mathrm{VI}]$} & 14.785 & $7.8: \pm 1.1$ & $0.27:$ \\
{$[\mathrm{Ne} \mathrm{III}]$} & 15.553 & $1370 \pm 70$ & 46.9 \\
{$[\mathrm{~S} \mathrm{III}]$} & 18.712 & $306 \pm 9$ & 10.5 \\
{$[\mathrm{Cl} \mathrm{IV}]$} & 20.312 & $21.5: \pm 0.5$ & $0.74:$ \\
{$[\mathrm{Ar} \mathrm{III}]$} & 21.819 & $11.7: \pm 1.1$ & $0.40:$ \\
{$[\mathrm{Ne} \mathrm{V}]$} & 24.319 & $1860 \pm 15$ & 63.7 \\
{$[\mathrm{O}$ IV] } & 25.887 & $8690 \pm 940$ & 298 \\
{$[\mathrm{~S} \mathrm{III}]$} & 33.477 & $149 \pm 7$ & 5.11 \\
{$[\mathrm{Si} \mathrm{II}]$} & 34.818 & $8.0: \pm 1.2$ & $0.275:$ \\
{$[\mathrm{Ne}$ III $]$} & 36.008 & $106 \pm 4$ & 3.63 \\
\hline
\end{tabular}

$\dagger$ Intensities measured in units of $10^{-14} \mathrm{erg} \mathrm{cm}^{-2} \mathrm{~s}^{-1}$. The intensities below $19 \mu \mathrm{m}$ have been increased by a factor of 2.65 and those by a factor of 5.5 to bring them all to the same scale (see text).

(:) Indicates an uncertain value.

Note: The measured line intensity is given in Col. 3. The last column gives the ratio of the line intensity to $\mathrm{H} \beta(=100)$.

\subsection{The IUE ultraviolet spectrum}

Only three IUE observations of NGC 2792 were made. Two were short-wavelength measurements, SWP16018 and SWP16032, and a long-wavelength measurement, LWR12326. All of them were made at the position $\mathrm{RA}(2000) 09^{\mathrm{h}} 12^{\mathrm{m}} 26.74^{\mathrm{s}}$ and $\operatorname{Dec}(2000)-42^{\circ} 25^{\prime} 31.6^{\prime \prime}$, which is about $8^{\prime \prime}$ from the center of the nebula, so that only part of the nebula was seen. In order to account for the missing radiation and for the extinction a correction was made in the short-wavelength region by assuming a theoretical ratio for the He II line ratio $\lambda 1640 / \lambda 4686 \AA$ at $T=15000 \mathrm{~K}$ and an $N_{\mathrm{e}}$ of $10^{3} \mathrm{~cm}^{-3}$. The ratio of $\lambda 1640$ to $\mathrm{H} \beta$ can then be found using the $\lambda 4686 / \mathrm{H} \beta$ ratio in Table 2 . A further correction for extinction relative to $\lambda 1640 \AA$ is then made using the reddening curve of Fluks et al. (1994) and a value of the extinction constant $C=0.80$ (see below). For the longwavelength region essentially the same procedure was carried out, the only difference being that an average value for the He II lines at $\lambda 2733 \AA$ and $\lambda 3204 \AA$ were used. This has the consequence that the correction for diaphragm size is somewhat different in the two wavelength regions, probably because the actual position of the measurements were slightly different. The value of the intensity of the C III $\lambda 1906 \AA$ line, which was measured in both wavelength regions, is found to have the same ratio with respect to $\mathrm{H} \beta$ in both regions. The uncertainties in the intensities are estimated to be $30 \%$ for the stronger lines and $50 \%$ for the weaker lines. The results are shown in the last two columns of Table 3, where the measured intensities are given in Col. 3, the intensities corrected for diaphragm size and for extinction are found in Col. 4 , and the last column gives the ratio of the intensity to $\mathrm{H} \beta$, normalized to $\mathrm{H} \beta=100$.
Table 2. Visual spectrum of NGC 2792.

\begin{tabular}{|c|c|c|c|c|c|c|}
\hline \multirow{2}{*}{$\begin{array}{c}\lambda \\
(\AA)\end{array}$} & \multirow[t]{2}{*}{ Ion } & \multicolumn{4}{|c|}{ Intensities $^{\dagger}$} & \multirow{2}{*}{$\begin{array}{l}\text { Average } \\
\text { Intens. }\end{array}$} \\
\hline & & (1) & (2) & (3) & (4) & \\
\hline 3426 & {$[\mathrm{Ne} \mathrm{V}]$} & & & 68: & 130: & 68 \\
\hline $3727^{*}$ & [O II] & 7.4: & & 14: & 12 & 9 \\
\hline 3869 & [Ne III] & 67.8 & & 68 & 63.1 & 67.8 \\
\hline 4267 & C II & 0.1: & & & & $0.1:$ \\
\hline 4363 & [O III] & 17.0 & 14.3 & 20 & 12.2 & 16 \\
\hline 4686 & He II & 97.0 & 101.3 & 91 & 100.4 & 98 \\
\hline 4711 & [Ar IV] & 9.7 & & & 8.73 & 9.2 \\
\hline 4740 & [Ar IV] & 8.5 & & & 7.94 & 8.1 \\
\hline 4861 & $\mathrm{H} \beta$ & 100 & 100 & 100 & 100 & 100 \\
\hline 5007 & [O III] & 960 & 1030 & 1150 & 1050 & 1050 \\
\hline 5517 & [Cl III] & 0.5 & & & & $0.5:$ \\
\hline 5538 & [Cl III] & 0.5 & & & & $0.5:$ \\
\hline 5755 & {$[\mathrm{~N} \mathrm{II}]$} & 0.1: & 1.0 & & & $0.4::$ \\
\hline 5876 & He I & 3.0 & 3.3 & 4.0: & 3.08 & 3.2 \\
\hline 6312 & [S III] & 1.8 & 1.8 & & & 1.8 \\
\hline 6584 & {$[\mathrm{~N} \mathrm{II}]$} & 3.5: & 10.4 & 4.4: & 5.26 & 4.4 \\
\hline 6717 & [S II] & 0.4 & & & & $0.4:$ \\
\hline 6731 & [S II] & 0.7 & & & & 0.7 : \\
\hline 7005 & [Ar V] & 3.2: & & & & 3.2: \\
\hline 7135 & [Ar III] & 9.3 & 8.2 & 10.7 & & 9.6 \\
\hline 7263 & [Ar IV] & 0.4 & & & & $0.4:$ \\
\hline 8045 & [Cl IV] & 1.6 & & & & 1.6 \\
\hline $\mathrm{C}(\mathrm{H} \beta)$ & & 0.67 & 1.08 & 0.75 & 1.05 & \\
\hline
\end{tabular}

$\dagger$ References; (1) Milingo et al. (2002a), (2) de Freitas Pacheco et al. (1992), (3) Torres-Peimbert\& Peimbert (1977), (4) Kingsburgh \& Barlow (1994).

(:) Indicates uncertain values.

* This is a blend of $\lambda 3726$ and $\lambda 3729$ lines. $C$ is the extinction used by the author.

Table 3. IUE spectrum of NGC 2792.

\begin{tabular}{clccc}
\hline \hline$\lambda$ & Ion & \multicolumn{3}{c}{ Intensities } \\
\cline { 3 - 5 }$(\AA)$ & & $(1)$ & $(2)$ & $(I / \mathrm{H} \beta)$ \\
\hline 1400 & O IV & 2.47 & 3.69 & 119 \\
1485 & N IV] & 1.89 & 2.44 & 79 \\
1523 & & 1.25 & 1.60 & 52 \\
1548 & C IV & 7.0 & 8.97 & 290 \\
1640 & He II & 18.1 & 21.8 & 704 \\
1663 & O III] & 1.83 & 2.13 & 69 \\
1750 & N III] & 1.74 & 1.86 & 60 \\
1887 & Si III] & 0.40 & 0.46 & $15:$ \\
1909 & C III] & 13.3 & 16.1 & 520 \\
2089 & & 0.75 & 2.51 & 81 \\
2423 & [Ne IV] & 5.13 & 8.84 & 274 \\
2456 & & 0.61 & 1.00 & 32 \\
2733 & He II & 1.09 & 0.86 & 26.8 \\
2831 & [Fe IV]? & 1.02 & 0.71 & 22 \\
$3043 / 47$ & O III & 1.6 & 0.93 & 29 \\
3133 & O III & 7.6 & 4.35 & 135 \\
3203 & He II & 2.55 & 1.19 & 37.0 \\
\hline
\end{tabular}

(1) Measured intensity from in units of $10^{-13} \mathrm{erg} \mathrm{cm}^{-2} \mathrm{~s}^{-1}$. (2) Intensity corrected for diaphragm size and extinction in units of $10^{-11} \mathrm{erg} \mathrm{cm}^{-2} \mathrm{~s}^{-1} . I / \mathrm{H} \beta$ is normalized to $\mathrm{H} \beta=100$. ":" Indicates uncertain value

\subsection{Extinction}

The two methods which can be used for obtaining the extinction are: (1) comparison of radio emission with $\mathrm{H} \beta$ flux, and (2) comparison of observed and theoretical Balmer decrement. The four values of the extinction correction $C(\mathrm{H} \beta)$ which are found in the 


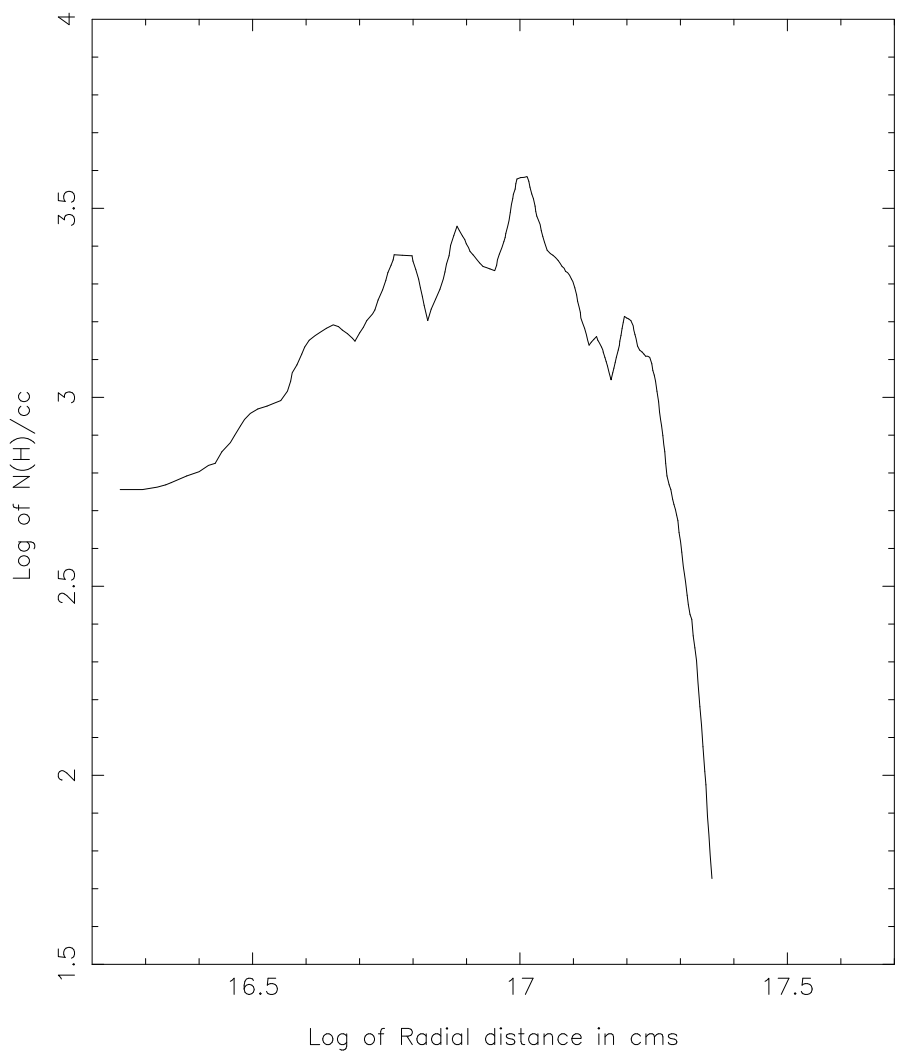

Fig. 3. The input density profile. The ordinate is the electron density.

literature are given in Table 2, and are seen to have a rather large range. Let us discuss the radio emission and the $\mathrm{H} \beta$ flux.

\subsubsection{The $6 \mathrm{~cm}$ radio emission and the $\mathrm{H} \beta$ flux}

The $6 \mathrm{~cm}$ flux density has been measured by Gregory et al. (1994) as $127 \mathrm{mJy}$ and by Wright et al. (1994) as $139 \mathrm{mJy}$. We use an average value of $133 \mathrm{mJy}$. This implies an $\mathrm{H} \beta$ flux of $3.05 \times 10^{-11} \mathrm{erg} \mathrm{cm}^{-2} \mathrm{~s}^{-1}$. Given an observed $\mathrm{H} \beta$ flux of $4.86 \times 10^{-12} \mathrm{erg} \mathrm{cm}^{-2} \mathrm{~s}^{-1}$ (see Cahn et al. 1992) yields an extinction constant of $C(\mathrm{H} \beta)=0.80$ or $E_{B-V}=0.55$. This is quite similar to the average value found from the Balmer decrement and listed in Table 2. This value will be used when necessary in the remainder of this paper together with the extinction curve of Fluks et al. (1994).

\section{Chemical composition of NGC 2792 from simplified analysis}

The method of analysis is the same as used in the papers cited in the introduction. First the electron density and temperature as function of the ionization potential are determined. Then the ionic abundances are determined, using density and temperature appropriate for the ion under consideration, together with Eq. (1). Then the element abundances are found for those elements in which a sufficient number of ion abundances have been derived.

\subsection{Electron density}

The ions used to determine $N_{\mathrm{e}}$ are listed in the first column of Table 4. The ionization potential required to reach that ionization stage, and the wavelengths of the lines used, are given in Cols. 2
Table 4. Electron density indicators in NGC 2792.

\begin{tabular}{lcccc}
\hline \hline Ion & $\begin{array}{c}\text { Ioniz. } \\
\text { Pot.(eV) }\end{array}$ & $\begin{array}{c}\text { Lines } \\
\text { Used }\end{array}$ & $\begin{array}{c}\text { Observed } \\
\text { Ratio }\end{array}$ & $\begin{array}{c}N_{\mathrm{e}} \\
\left(\mathrm{cm}^{-3}\right)\end{array}$ \\
\hline$[\mathrm{S} \mathrm{II}]$ & 10.4 & $6731 / 6716$ & $1.7:$ & $4500:$ \\
{$[\mathrm{S} \mathrm{III}]$} & 23.3 & $33.5 / 18.7$ & 0.50 & 2300 \\
{$[\mathrm{Cl}$ III $]$} & 23.8 & $5538 / 5518$ & $0.9:$ & $1500:$ \\
{$[\mathrm{Ar} \mathrm{IV}]$} & 40.7 & $4740 / 4711$ & 0.88 & 1500 \\
{$[\mathrm{Ar} \mathrm{V}]$} & 59.8 & $13.1 / 7.90$ & 1.35 & 4100 \\
{$[\mathrm{Ne} \mathrm{V}]$} & 97.1 & $24.3 / 14.3$ & 0.70 & 3000 \\
\hline
\end{tabular}

(:) Indicates uncertain values.

Table 5. Electron temperature indicators in NGC 2792.

\begin{tabular}{lcccc}
\hline \hline Ion & $\begin{array}{c}\text { Ioniz. } \\
\text { Pot.(eV) }\end{array}$ & $\begin{array}{c}\text { Lines } \\
\text { Used }\end{array}$ & $\begin{array}{c}\text { Observed } \\
\text { Ratio }\end{array}$ & $\begin{array}{c}T_{\mathrm{e}} \\
(\mathrm{K})\end{array}$ \\
\hline$[\mathrm{S} \mathrm{III}]$ & 23.3 & $6312 / 18.7$ & 0.171 & 16500 \\
{$[\mathrm{Ar} \mathrm{III}]$} & 27.6 & $7135 / 21.8$ & 24.0 & 13000 \\
{$[\mathrm{O}$ III $]$} & 35.1 & $4363 / 5007$ & 0.0177 & 14000 \\
{$[\mathrm{O} \mathrm{III}]$} & 35.1 & $1663 / 5007$ & 0.0557 & 15800 \\
{$[\mathrm{Ne} \mathrm{III}]$} & 41.0 & $3868 / 15.5$ & 1.45 & 13800 \\
{$[\mathrm{O} \mathrm{IV}]$} & 54.9 & $1400 / 25.9$ & 0.40 & 20000 \\
{$[\mathrm{Ne} \mathrm{v}]$} & 97.1 & $3425 / 24.3$ & 1.0 & 20000 \\
\hline
\end{tabular}

and 3 of the table. Note that the wavelength units are $\AA$ when 4 ciphers are given and microns when 3 ciphers are shown. The observed ratio of the lines is given in the fourth column; the corresponding $N_{\mathrm{e}}$ is given in the fifth column. The temperature used is discussed in the following section, but is unimportant since these line ratios are essentially determined by the density.

The electron density appears to be about 2000 to $3000 \mathrm{~cm}^{-3}$ but the value is not well determined because several of the measured ratios are poorly measured. There is no indication that the electron density varies with ionization potential in a systematic way. It is interesting to compare this value of the density with the rms density found from the $\mathrm{H} \beta$ line. This depends on the distance of the nebula which isn't accurately known, and on the angular size of the nebula. If a distance of $1.5 \mathrm{kpc}$ is used together with an angular radius of 5.5" an uncertain rms density of $1200 \mathrm{~cm}^{-3}$ is found. The density is probably not uniform, which together with the uncertain measurements is the cause of this difference. A density of $2500 \mathrm{~cm}^{-3}$ is used in the abundance determination in Table 6, but none of the abundances listed in the table is sensitive when the density is in the above range.

\subsection{Electron temperature}

A number of ions have lines originating from energy levels far enough apart that their ratio is sensitive to the electron temperature. These are listed in Table 5, which is arranged similarly to the previous table. The electron temperature seems to increase as a function of ionization potential. There is some scatter when these values are plotted as function of the ionization potential. No temperature is given for the [N II] lines because only two of the four spectra showed the $\lambda 5755 \AA$ line and these two measurements gave very different intensities for the line. The $\mathrm{Ne} \mathrm{V}$ temperature is very uncertain because the intensity of the line at $\lambda 3425 \AA$ is badly determined. From the above plot we are able to determine a temperature for each ion considered. For an extension of the plot to low values of ionization potential we have relied on information given by the model. Because of uncertainty of the electron temperature due to scatter we have given more 
weight to the abundances found from the infrared lines which are insensitive to the electron temperature.

\subsection{Ionic and element abundances}

The ionic abundances have been determined using the following equation:

$\frac{N_{\text {ion }}}{N_{\mathrm{p}}}=\frac{I_{\text {ion }}}{I_{\mathrm{H}_{\beta}}} N_{\mathrm{e}} \frac{\lambda_{\mathrm{ul}}}{\lambda_{\mathrm{H}_{\beta}}} \frac{\alpha_{\mathrm{H}_{\beta}}}{A_{\mathrm{ul}}}\left(\frac{N_{\mathrm{u}}}{N_{\text {ion }}}\right)^{-1}$

where $I_{\text {ion }} / I_{\mathrm{H}_{\beta}}$ is the measured intensity of the ionic line compared to $\mathrm{H} \beta, N_{\mathrm{p}}$ is the density of ionized hydrogen, $\lambda_{\mathrm{ul}}$ is the wavelength of this line, $\lambda_{\mathrm{H}_{\beta}}$ is the wavelength of $\mathrm{H} \beta, \alpha_{\mathrm{H}_{\beta}}$ is the effective recombination coefficient for $\mathrm{H} \beta, A_{\mathrm{ul}}$ is the Einstein spontaneous transition rate for the line, and $N_{\mathrm{u}} / N_{\text {ion }}$ is the ratio of the population of the level from which the line originates to the total population of the ion. This ratio has been determined using a five level atom. For Si, since the Si III UV line measurement is very uncertain, we have used only the IR line.

The results are given in Table 6, where the first column lists the ion concerned, and the second column the line used for the abundance determination. The third column gives the intensity of the line used relative to $\mathrm{H} \beta=100$. The fourth column gives the temperature used for the ion, the fifth column shows the ionic abundances, and the sixth column gives the Ionization Correction Factor (ICF). This has been determined empirically, usually by looking at the ionization potential of the missing ion. Notice that the ICF is unity for all elements except for Ar, S and $\mathrm{Cl}$ where it is close to unity. The exception to this is for $\mathrm{Si}$ and $\mathrm{Mg}$ where only single ionization stages are measured. In these two cases the ICF is estimated with the help of the model described below. In the other cases the element abundances, given in the last column, are probably well determined. The abundance of carbon is more uncertain than the other elements because both, the $\mathrm{C}$ III] and $\mathrm{C}$ IV lines, are quite sensitive to the electron temperature.

\section{Model}

In order to obtain as nearly a correct model as possible, the star as well as the nebula must be considered. Modeling the nebulastar complex will allow characterizing not only the central star's temperature but other stellar parameters as well (i.e., log g and luminosity). It can determine distance and other nebular properties, especially the composition, including the composition of elements that are represented by a single stage of ionization, which cannot be determined by the simplified analysis above. This method can take the presence of dust and molecules into account in the nebular material, when there is any there, making it a very comprehensive approach. While the line ratio method is simple and fast, the ICFs rest on uncertain physics. To this end, modeling serves as an effective means, and the whole set of parameters are determined in a unified way, assuring self consistency. Also, in this way one gets good physical insight into the PN, the method and the observations. Thus, modeling is a good approach to an end-to-end solution to the problem.

It is with this in mind that we have constructed a photoionization model for NGC 2792 with the code Cloudy, using the latest version C08.00 of Aug. 2008. (Ferland et al. 1998). Information on the updates in this version can be found on Cloudy's website.
Table 6. Ionic concentrations and chemical abundances in NGC 2792.

\begin{tabular}{|c|c|c|c|c|c|c|}
\hline Ion & $\lambda$ & $\begin{array}{l}\text { Int./H } \beta \\
\end{array}$ & $T$ & $N_{\text {ion }} / N_{\mathrm{p}}$ & ICF & $N_{\mathrm{el} .} / N_{\mathrm{p}}$ \\
\hline $\mathrm{He}^{+}$ & 5875 & 3.0 & 15000 & 0.017 & & \\
\hline $\mathrm{He}^{++}$ & 4686 & 97 & 16000 & 0.0855 & 1 & 0.103 \\
\hline $\mathrm{C}^{++}$ & 1909 & 520 & 14500 & $9.5(-5)$ & & \\
\hline $\mathrm{C}^{+3}$ & 1548 & 290 & 17000 & $1.5(-5)$ & 1 & $1.1(-4)$ \\
\hline $\mathrm{N}^{+}$ & 6584 & 4.4: & 13000 & $4.0(-7)$ & & \\
\hline $\mathrm{N}^{++}$ & 1750 & 60.1 & 15500 & $4.1(-5)$ & & \\
\hline $\mathrm{N}^{+3}$ & 1485 & 79 & 17000 & $2.6(-5)$ & 1 & $6.8(-5)$ \\
\hline $\mathrm{O}^{+}$ & 3727 & 9 & 13000 & $1.8(-6)$ & & \\
\hline $\mathrm{O}^{++}$ & 5007 & 960 & 15000 & $1.03(-4)$ & & \\
\hline $\mathrm{O}^{++}$ & 1663 & 69 & 15000 & $1.50(-4)$ & & \\
\hline $\mathrm{O}^{+3}$ & 25,9 & 298 & 18000 & $7.7(-5)$ & 1 & $2.1(-4)$ \\
\hline $\mathrm{Ne}^{+}$ & 12.8 & 1.1 & 15000 & $1.1(-6)$ & & \\
\hline $\mathrm{Ne}^{++}$ & 15.5 & 46.9 & 16000 & $2.46(-5)$ & & \\
\hline $\mathrm{Ne}^{++}$ & 3869 & 67.8 & 16000 & $1.44(-5)$ & & \\
\hline $\mathrm{Ne}^{+3}$ & 2424 & 191.0 & 18000 & $3.29(-5)$ & & \\
\hline $\mathrm{Ne}^{+4}$ & 14.3 & 92.6 & 18000 & $5.8(-6)$ & 1 & $6.5(-5)$ \\
\hline $\mathrm{S}^{+}$ & 6731 & 1.8 & 13000 & $5.0(-8)$ & & \\
\hline $\mathrm{S}^{++}$ & 6312 & 1.8 & 15000 & $7.7(-7)$ & & \\
\hline $\mathrm{S}^{++}$ & 18.7 & 10.5 & 15000 & $8.04(-7)$ & & \\
\hline $\mathrm{S}^{+3}$ & 10.5 & 108 & 16000 & $2.37(-6)$ & 1.3 & $4.3(-6)$ \\
\hline $\mathrm{Ar}^{++}$ & 8.99 & 8.06 & 15000 & $6.11(-7)$ & & \\
\hline $\mathrm{Ar}^{++}$ & 7135 & 9.3 & 15000 & $3.6(-7)$ & & \\
\hline $\mathrm{Ar}^{+3}$ & 4740 & 8.2 & 16000 & $6.3(-7)$ & & \\
\hline $\mathrm{Ar}^{+4}$ & 7005 & 3.2 & 18000 & $2.2(-7)$ & & \\
\hline $\mathrm{Ar}^{+4}$ & 7.90 & 5.96 & 18000 & $2.04(-7)$ & 1.1 & $1.6(-6)$ \\
\hline $\mathrm{Cl}^{++}$ & 5538 & 0.5 & 15000 & $2.4(-8)$ & & \\
\hline $\mathrm{Cl}^{+3}$ & 11.8 & 0.894 & 16000 & $4.0(-8)$ & 1.4 & $0.90(-7)$ \\
\hline $\mathrm{Mg}^{+4}$ & 5.60 & 11.6 & 18000 & $2.3(-6)$ & 4: & 1.0(-5): \\
\hline $\mathrm{Si}^{+}$ & 34.8 & 0.275 & 13000 & $1.1(-7)$ & 20: & $2(-6):$ \\
\hline
\end{tabular}

Intensities given with respect to $\mathrm{H} \beta=100$. (:) Indicates uncertain values.

Note: Wavelength in Angstrom for all values of $\lambda$ above 1000, otherwise in $\mu \mathrm{m}$.

\subsection{Assumptions}

Looking at the HST visual image shown in Fig. 1, it is reasonable to assume that the nebula is nearly spherical. We then determined its angular radius as 10 arcsec from this image. Initially we also assumed in the model that the spatial variation of density is minimal so a constant density model would be sufficient. But later, after some trial runs, we thought it appropriate to use a model in which the density varies radially across the nebula. The HST image being a broadband visual one, $\mathrm{H}$-alpha images give a better estimate of the actual density $(N(\mathrm{H}) / \mathrm{cc})$. Therefore we used the $\mathrm{H}$-alpha image of this PN from http://astro.uni-tuebingen.de/groups/pn/. This image was downloaded and used as is, since no additional information was available about it. We could import it into the IRAF facility and about 30 cross-cuts (i.e., density profiles) were taken over several clock positions and averaged. It should be noted that at this stage this average profile represents neither an emission measure nor true density. It is simply a count vs. pixel profile since the original image does not have any information on whether it is fully processed and intensity calibrated. This profile was then smoothed, used as a template, and converted to density vs angular radius using a radius of 10 arcsec for the nebula, since the template only gives counts vs pixels. The counts were then normalized to $N(\mathrm{H}) / \mathrm{cc}$ assuming a nominal value of $3000 / \mathrm{cc}$ for the peak of the profile. Later in the actual modeling process, we not only allowed the distance to be a free parameter (affecting the radial profile since any change in distance changes the linear radius) but also varied the density by enhancing or decreasing the 
Table 7. Parameters representing the final model.

\begin{tabular}{|c|c|c|c|c|}
\hline Parameter & \multicolumn{4}{|l|}{ Value } \\
\hline Ionizing source & & & & \\
\hline Model atmosphere & \multicolumn{4}{|c|}{ Rauch H-Ni } \\
\hline$T_{\text {eff }}$ & \multicolumn{4}{|c|}{$160000 \mathrm{~K}$} \\
\hline $\log g$ & \multicolumn{4}{|c|}{6.00} \\
\hline Luminosity & \multicolumn{4}{|l|}{$600 L_{\odot}$} \\
\hline \multicolumn{5}{|l|}{ Nebula } \\
\hline Density profile & \multicolumn{4}{|c|}{ See Fig. 3} \\
\hline \multirow{6}{*}{ Abundance } & $\mathrm{H}$ & $\mathrm{He}$ & $\mathrm{C}$ & $\mathrm{N}$ \\
\hline & 12.000 & 11.000 & 7.900 & 7.641 \\
\hline & $\mathrm{O}$ & $\mathrm{Ne}$ & $\mathrm{Mg}$ & $\mathrm{Si}$ \\
\hline & 8.321 & 7.799 & 7.031 & 6.455 \\
\hline & & $\mathrm{Cl}$ & $\mathrm{Ar}$ & \\
\hline & 6.648 & 5.049 & 6.213 & \\
\hline Size & \multicolumn{4}{|c|}{$10^{\prime \prime}$ (radius) } \\
\hline Distance & \multicolumn{4}{|c|}{$1.50 \mathrm{kpc}$} \\
\hline Dust grains & \multirow{2}{*}{\multicolumn{4}{|c|}{$\begin{array}{l}\text { Silicates of single size } 1.0 \mu \mathrm{m} \text {; } \\
2.244 \mathrm{e} 16 \mathrm{~cm}\end{array}$}} \\
\hline $\begin{array}{l}\text { inner radius } \\
\text { outer radius }\end{array}$ & & & & \\
\hline Filling factor & \multicolumn{4}{|c|}{1.0} \\
\hline
\end{tabular}

actual $N(\mathrm{H}) / \mathrm{cc}$ value at each radial point. The profile used in the final model is shown in Fig. 3. Because determinating the density is difficult using line ratios, the above method was thought to be the best alternative. We have included dust grains mixed with gas in the nebula, so that we could compare model dust grain emission continua with the Spitzer observations. To represent the central star's ionizing radiation, we experimented with several model atmospheres available within the built-in library of Cloudy. We also tried pure black-body radiation in some test models.

\subsection{Results}

The general method of applying the code Cloudy to model NGC 2792 is the same as in Surendiranath et al. (2004). We ran a number of models and each time the output was carefully scrutinized before running the next model with changed input parameters. We tried to match the observed spectral fluxes of about 70 lines and we relied on physical intuition rather than any of the optimization techniques provided in Cloudy. The results are presented in Tables 7 and 8 and discussed in more detail in the following section, where we treat the abundances determined by the empirical (ICF) method as our final values for this PN.

\section{Discussion}

After a number of models were computed as discussed in the previous section, it gradually became clear that it was very difficult to reproduce the observed emission line fluxes, especially those of He II, C III] in the UV and [O IV] in the IR region. The observed absolute $\mathrm{H} \beta$ flux could be reproduced well. See the final model output spectra in Table 8 . This was the best that could be done with the many models we tested. The corresponding input parameters are listed in Table 7. In the final model, we used the Rauch (2003) model atmosphere to represent the central star's ionizing radiation. We recommend the abundances as determined by the empirical line ratio method for this PN and discuss some important implications of our modeling exercise below.

\subsection{Possible causes for non-convergence of the model}

The basic problem is that none of our models tested could reproduce the apparently very high level of emitted flux in lines of certain ions mentioned above. Because of this high level of excitation we tried modeling with first a binary and then with a very high temperature star. As a binary for the CSPN we inputted the energies of two different model atmospheres together, but this did not help. We then tried a blackbody model atmosphere of $T_{\text {eff }}=300000 \mathrm{~K}$ and this also could not reproduce the line of He II and [O IV] among others. The diagnostic lines did not agree well with observed values. Changing other parameters also did not work. Our final model was the best we could achieve. One possibility for nonconvergence of the model output spectrum with the observations could be that the slits used to obtain the various spectra are different and the combining of these various spectra introduces errors which differ with the different lines. While this must play a small role, most of the observations cover a substantial part of the nebula, and, as already discussed, are always normalized to the same $\mathrm{H} \beta$. The optical measurements are generally made with a long slit which is about $5^{\prime \prime}$ wide and thus integrate about half of the nebular light. The infrared LH measurement covers an even larger region of the nebula (judging from the hydrogen lines more than $90 \%$ of the nebula is seen) but the infrared SH covers a smaller region. Finally the UV measurements cover only about one half of the nebula because they are not well centered. However because all measurements integrate along the line-of-sight we doubt that this is the cause of the discrepancy. Other explanations include the possibility that this PN has a central star that emits an unusual or peculiar energy spectrum, such that no currently available model atmosphere can represent it. While this cannot be ruled out it seems to us unlikely that such a hot star would deviate substantially from a blackbody. An alternative possibility is that the model's assumed geometry is wrong. We had assumed a spherical shell based on the visual image. The PN could possibly have a different geometry and still have a circular appearance because the axis of symmetry is in the line-of-sight. But no models with axi-symmetric geometry have been studied in the literature so the effect of a different geometry cannot be ascertained. Only dynamical studies will shed more light on a possible axi-symmetric geometry for the nebula.

The UV line at $\lambda 2831 \AA$, could be due to [Fe IV] but this assumption required a very high iron abundance and hence we doubt its identification with that ion. This is true with regard to the line ratio method as well. The final model's input and output are shown in tables only for the purpose of illustration of the difficulties faced. It reproduced both the absolute $\mathrm{H}_{\beta}$ flux (Table 8) and the grain emitted continuum in the IR (not shown here) very well.

\section{Comparison with other abundance determinations}

Table 9 shows a comparison of our abundances with the most important determinations in the past 20 years. The agreement is not very good, differences of a factor of three or more are common. The probable reasons for this are discussed in the following subsection. A comparison is also made with the solar abundance (Grevesse et al. 2007) and is given in the last column.

The helium abundance has been derived using the theoretical work of Benjamin et al. (1999) and Porter et al. (2005). For recombination of singly ionized helium, most weight is given to 
Table 8. The emission line fluxes $(\mathrm{H} \beta=100)$.

\begin{tabular}{|c|c|c|c|c|c|c|c|}
\hline Label & Line & Model flux & $\begin{array}{r}\text { Obsd. flux } \\
\text { (dereddened) }\end{array}$ & Label & Line & Model flux & $\begin{array}{r}\text { Obsd. flux } \\
\text { (dereddened) }\end{array}$ \\
\hline TOTL & $4861 \mathrm{~A}$ & 100.00 & 100.00 & Ar 5 & $7005 \mathrm{~A}$ & 4.97 & 3.20 \\
\hline TOTL & $1402 \mathrm{~A}$ & 82.62 & 119.20 & $\mathrm{He} 1$ & $7065 \mathrm{~A}$ & 1.39 & 0.80 \\
\hline TOTL & $1486 \mathrm{~A}$ & 83.97 & 79.00 & Ar 3 & $7135 \mathrm{~A}$ & 10.54 & 9.60 \\
\hline TOTL & $1549 \mathrm{~A}$ & 539.88 & 290.00 & Ar 4 & 7237A & 0.46 & 0.40 \\
\hline He 2 & $1640 \mathrm{~A}$ & 589.85 & 704.00 & Ar 4 & $7263 \mathrm{~A}$ & 0.51 & 0.40 \\
\hline TOTL & $1665 \mathrm{~A}$ & 72.48 & 69.00 & O II & 7323A & 0.75 & 0.60 \\
\hline TOTL & $1750 \mathrm{~A}$ & 56.40 & 60.00 & $\mathrm{Cl} 4$ & $7532 \mathrm{~A}$ & 0.39 & 0.70 \\
\hline TOTL & 1909A & 466.75 & 520.00 & He 2 & 7593A & 1.23 & 1.20 \\
\hline $\mathrm{Ne} 4$ & $2424 \mathrm{~A}$ & 226.27 & 274.00 & Ar 3 & 7751A & 2.54 & 1.80 \\
\hline He 2 & $2733 \mathrm{~A}$ & 18.33 & 26.80 & $\mathrm{Cl} 4$ & 8047A & 0.78 & 1.60 \\
\hline He 2 & $3203 \mathrm{~A}$ & 34.62 & 37.00 & He 2 & 8237A & 1.73 & 2.00 \\
\hline TOTL & 3727A & 14.58 & 7.40 & H 1 & $9015 \mathrm{~A}$ & 1.68 & 1.50 \\
\hline $\mathrm{Ne} 3$ & 3869A & 137.51 & 67.80 & S 3 & 9069A & 15.81 & 6.50 \\
\hline $\mathrm{Ne} 3$ & 3968A & 41.44 & 38.80 & H 1 & 9229A & 2.32 & 2.50 \\
\hline S II & 4070A & 0.71 & 1.10 & S 3 & $9532 \mathrm{~A}$ & 39.30 & 19.20 \\
\hline C 2 & $4267 \mathrm{~A}$ & 0.04 & 0.10 & H 1 & 9546A & 3.35 & 2.60 \\
\hline H 1 & $4340 \mathrm{~A}$ & 47.28 & 50.30 & $\operatorname{Mg} 5$ & $5.610 \mathrm{~m}$ & 10.67 & 11.60 \\
\hline TOTL & $4363 \mathrm{~A}$ & 28.06 & 16.00 & $\operatorname{Ar} 5$ & $8.000 \mathrm{~m}$ & 7.01 & 6.00 \\
\hline He 1 & $4471 \mathrm{~A}$ & 1.14 & 0.70 & Ar 3 & $9.000 \mathrm{~m}$ & 4.34 & 8.10 \\
\hline He 2 & $4686 \mathrm{~A}$ & 86.60 & 98.00 & S 4 & $10.51 \mathrm{~m}$ & 147.23 & 108.00 \\
\hline $\operatorname{Ar} 4$ & $4740 \mathrm{~A}$ & 14.39 & 8.50 & $\mathrm{Cl} 4$ & $11.70 \mathrm{~m}$ & 1.22 & 0.89 \\
\hline O 3 & 4959A & 358.34 & 316.60 & $\mathrm{Ne} 2$ & $12.81 \mathrm{~m}$ & 0.12 & 0.99 \\
\hline O 3 & $5007 \mathrm{~A}$ & 1078.60 & 1050.00 & Ar 5 & $13.10 \mathrm{~m}$ & 10.01 & 7.07 \\
\hline He 2 & $5412 \mathrm{~A}$ & 6.24 & 8.40 & $\operatorname{Mg} 5$ & $13.52 \mathrm{~m}$ & 0.89 & 0.66 \\
\hline $\mathrm{Cl} 3$ & 5518A & 0.77 & 0.50 & $\mathrm{Ne} 5$ & $14.32 \mathrm{~m}$ & 118.81 & 92.60 \\
\hline $\mathrm{Cl} 3$ & $5538 \mathrm{~A}$ & 0.75 & 0.50 & $\mathrm{Ne} 3$ & $15.55 \mathrm{~m}$ & 46.90 & 46.90 \\
\hline O 1 & 5577A & 0.00 & 0.20 & S 3 & $18.67 \mathrm{~m}$ & 12.52 & 10.50 \\
\hline N 2 & $5755 \mathrm{~A}$ & 0.17 & 0.10 & $\mathrm{Cl} 4$ & $20.40 \mathrm{~m}$ & 1.13 & 0.74 \\
\hline He 1 & $5876 \mathrm{~A}$ & 3.46 & 3.20 & Ar 3 & $21.83 \mathrm{~m}$ & 0.32 & 0.40 \\
\hline S 3 & $6312 \mathrm{~A}$ & 2.72 & 1.80 & Ne 5 & $24.31 \mathrm{~m}$ & 106.08 & 63.70 \\
\hline N 2 & $6548 \mathrm{~A}$ & 1.44 & 1.90 & O 4 & $25.88 \mathrm{~m}$ & 387.78 & 298.00 \\
\hline N 2 & $6584 \mathrm{~A}$ & 4.24 & 3.50 & S 3 & $33.47 \mathrm{~m}$ & 8.02 & 5.11 \\
\hline He 1 & 6678A & 0.80 & 1.40 & Si 2 & $34.81 \mathrm{~m}$ & 0.26 & 0.28 \\
\hline S II & $6716 \mathrm{~A}$ & 1.64 & 0.40 & $\mathrm{Ne} 3$ & $36.01 \mathrm{~m}$ & 4.10 & 3.63 \\
\hline S II & $6731 \mathrm{~A}$ & 2.16 & 0.70 & & & & \\
\hline
\end{tabular}

$\dagger$ Wavelength in Angstroms.

Absolute $\mathrm{H} \beta$ flux Model: $3.06 \times 10^{-11} \mathrm{ergs} \mathrm{cm}^{-2} \mathrm{~s}^{-1}$ Obsn: $3.10 \times 10^{-11} \mathrm{ergs} \mathrm{cm}^{-2} \mathrm{~s}^{-1}$.

Notes: "A" in Col. "Line" signifies Angstrom; "m" signifies $\mu \mathrm{m}$. In Col. "Label", we have followed the notation used by Cloudy for atoms and ions; this will make identifying a line in Cloudy's huge line list easy. Neutral state is indicated by "1" and singly ionized state by " 2 " etc., "TOTL" typically means the sum of all the lines in the doublet/multiplet; or it could mean sum of all processes: recombination, collisional excitation, and charge transfer. Some elements are represented by usual notation as per Cloudy.

the $\lambda 5875 \AA$ line, because the theoretical determination of this line is the most reliable.

\subsection{Errors}

It is difficult to determine the errors in the abundance determination. The reason for this is the following. The error can occur at several stages in the determination. An error can occur in the intensity determination and this can be specified: it is probably less than $30 \%$ and may be lower for the stronger lines. An error may occur in correcting for the extinction, either because the extinction is incorrect or the average reddening law is not applicable. We have tried to minimize this possibility by making use of known atomic constants to relate the various parts of the spectrum. Thus the ratio of the infrared spectrum to the visible spectrum is fixed by the ratio of infrared hydrogen lines to $\mathrm{H} \beta$ which is essentially an atomic constant.

In Table 1 the measurements of three hydrogen lines are given as well as the observed ratio of these lines to $\mathrm{H} \beta$. We may compare these observed ratios with the theoretical ratios for an electron temperature of $15000 \mathrm{~K}$. Because the $12.368 \mu \mathrm{m}$ line is used to derive the observed $\mathrm{H} \beta$ the ratio automatically agrees. The $7.478 \mu \mathrm{m}$ line which measures the 6-5, 8-6 and 17-8 transitions is expected to have a ratio of 2.67 compared to the $14 \%$ higher observed value of 3.05 . The $11.309 \mu \mathrm{m}$ line is expected to have a value of 0.253 whereas the observed value is 0.33 . While this is a difference of $30 \%$ the line is among the weakest observed and thus the highest expected error.

\subsection{Reasons for the difference}

The most important reason that the abundances we obtain differ from the earlier results in the literature is our ability to recognize that a gradient of the electron temperature exists in the nebula. This is easiest to see in the case of oxygen. All the earlier results give an electron temperature from the O III line ratio in the visual spectrum. Values are found for the temperature between 12000 and $14200 \mathrm{~K}$. From the same lines we find a similar value, between $14000 \mathrm{~K}$ and $15000 \mathrm{~K}$ (see Table 5). Consequently we find a value of the $\mathrm{O}$ III abundance which is 
Table 9. Comparison of abundances in NGC 2792.

\begin{tabular}{llllllll}
\hline \hline Ele. & Empir. & Model & HKB & KB & TPP & dFP & Solar \\
\hline He & 0.103 & 0.10 & 0.11 & 0.098 & 0.107 & 0.115 & 0.098 \\
$\mathrm{C}(-4)$ & 1.1 & 0.8 & & 8.4 & & & 2.5 \\
$\mathrm{~N}(-5)$ & 6.8 & 4.37 & 14 & $36:$ & $8.0:$ & & 6.0 \\
$\mathrm{O}(-4)$ & 2.1 & 2.1 & 7.65 & 5.74 & $6.0:$ & 5.6 & 4.6 \\
$\mathrm{~S}(-6)$ & 4.3 & 4.45 & 5.3 & & & & 14 \\
$\mathrm{Ar}(-6)$ & 1.6 & 1.63 & 6.4 & 2.2 & & 1.7 & 4.2 \\
$\mathrm{Ne}(-5)$ & 7.4 & 6.3 & 12 & 12.9 & $9.5:$ & & 12 \\
$\mathrm{Cl}(-7)$ & 0.90 & 1.12 & 5.2 & & & & 3.5 \\
$\mathrm{Si}(-6)$ & $2.0:$ & 2.85 & & & & & 32 \\
$\mathrm{Mg}(-5)$ & $1.0:$ & 1.07 & & & & & 3.4 \\
\hline
\end{tabular}

References: HKB: Henry et al. (2005), KB: Kingsburgh \& Barlow (1994), TPP: Torres-Peimbert \& Peimbert (1977), dFP: de Freitas Pacheco et al. (1992), Solar: Grevesse et al. (2007). The numbers in parentheses are the powers of ten used in the other columns.

similar to the earlier results. The difference in the total oxygen abundance occurs because in the earlier work this same temperature is used to determine the O IV abundance from the UV line at $1400 \AA$ which gives a very high abundance for this ion. We are able to use the infrared O IV line, which is almost temperature insensitive, to determine the O IV abundance and find that it really has a much lower value. The high nitrogen and carbon abundance found by Kingsburgh \& Barlow (1994) has the same origin: too low an electron temperature was used in conjunction with the ultraviolet lines of N III, N IV, C III and C IV, which are all very temperature-sensitive. This emphasizes the importance of using the temperature insensitive infrared lines. Notice that our results demonstrate that the carbon abundance is only about half of the oxygen abundance, while the work of Kingsburgh \& Barlow (1994) find carbon to be 50\% higher than oxygen.

A further error may be introduced by the correction for unseen stages of ionization. This varies with the element, but is usually small because very many ionization stages are observed. When the model is included the error due to missing stages of ionization becomes quite small.

\section{Stellar evolution}

The modeling exercise provides some clues regarding the nature of the stellar energy distribution and the stellar luminosity and effective temperature (see Table 7). These values may be compared to more conventional methods. The $T_{\text {eff }}$ found from the Zanstra method by Gathier \& Pottasch (1988) gives a hydrogen Zanstra temperature of $82000 \mathrm{~K}$ and a HeII Zanstra temperature of $135000 \mathrm{~K}$, using a magnitude $V=17.04$. The Energy Balance method using the line intensities in the first three tables of this paper and the theory of Preite-Martinez \& Pottasch (1983) leads to a temperature of about $100000 \mathrm{~K}$. It is rather difficult to deduce a precise value of the temperature. Ignoring the hydrogen Zanstra temperature because the nebula may be optically thin to ionizing radiation, a reasonable, though uncertain, average value is $T_{\text {eff }}=130000 \mathrm{~K}$. Using this value of temperature and the above stellar magnitude and distance, the stellar radius is $0.068 R_{\odot}$. Combined with the above temperature, the luminosity of the star is $1.2 \times 10^{3} L_{\odot}$. This is a factor of 2 higher than the value used in the modeling and is listed in Table 7 . These two values have been determined independently of each other, and considering the uncertainties in their determination they agree within a factor of two.

The abundances in NGC 2792 are similar to the solar abundance and to other $\mathrm{PNe}$ which are thought to originate from low mass stars. The abundances are almost identical to those of IC 2448 (Guiles et al. 2007), the only differences larger than a factor of two are the lower carbon and higher sulfur abundances in NGC 2792. Other PNe with similar abundances are NGC 6210, NGC 6369 and NGC 7662. All these nebulae have a low helium abundance, similar to the Sun, as well as nitrogen abundances whose low values indicate that very little nitrogen has been formed in the course of evolution. This is in agreement with theoretical calculations of the evolution of stars whose initial mass is close to $1 M_{\odot}$, e.g. Karakas (2003).

\section{Conclusions}

New IR spectra of NGC 2792 from the Spitzer Space Telescope have been presented and are analyzed in conjunction with other archival spectra in the UV and optical range. The abundances of $\mathrm{He}, \mathrm{C}, \mathrm{N}, \mathrm{O}, \mathrm{S}, \mathrm{Ar}, \mathrm{Ne}$ and $\mathrm{Cl}$ are found by the empirical method.

We have attempted a photoionization model for this nebula and found that the observed spectra could not be reproduced fully satisfactorily and hence recommend the abundances determined by the empirical method although the differences are small. Abundances of $\mathrm{Si}$ and $\mathrm{Mg}$ were found using the ionization levels found by the model. We have speculated on possible causes for the non-convergence of the model which could be either because the central star radiation is difficult to determine (i.e., non-representable by current model atmospheres) or because the geometry of the nebula complicated (i.e., it may be bipolar) and hence spherical symmetry is non-applicable. The central star's parameters became difficult to determine from the model because of this. Nevertheless the model is useful in two ways. First, when used together with the observed spectrum it provides insight into the electron temperature gradient in the nebula. Second, it makes it possible to correct for the missing ionization stages, especially in the cases of $\mathrm{Mg}$ and $\mathrm{Si}$, and thus makes meaningful abundance determinations possible for these elements.

The abundances found have been compared with those previously determined for this nebula. We show that rather large differences exist and are due largely to neglect of being able to recognize electron temperature gradients in the nebula in previous work.

The central star is also discussed. The temperature and luminosity of the star have been determined. Together with the nebular abundances we show that this star evolved from an initial mass close to $1 M_{\odot}$.

Acknowledgements. We duly acknowledge the use of SIMBAD and ADS in this research work. We have used the IUE spectra archive at the STSCI and we wish to thank the archive unit for the same.

\section{References}

Benjamin, R. A., Skillman, E. D., \& Smits, D. P. 1999, ApJ, 514, 307 Bernard Salas, J., Pottasch, S. R., Beintema, D. A., \& Wesselius, P. R. 2001, A\&A, 367, 949

Cahn, J. H., Kaler, J. B., \& Stanghellini, L. 1992, A\&AS, 94, 399 Corradi, R. L. M., Schonberner, D., Steffen, M., et al. 2003, MNRAS, 340, 417 Condon, J. J., \& Kaplan, D. L. 1998, ApJS 117, 361

Davey, A. R., Storey, P. J., \& Kisielius, R. 2000, A\&AS, 142, 85 Ferland, G. J., Korista, K. T., Verner, D. A., et al. 1998, PASP, 110, 761 Fluks, M. A., Plez, B., de Winter, D., et al. 1994, A\&AS, 105, 311 de Freitas Pacheco, J. A., Maciel, W. J., \& Costa, R. D. D. 1992, A\&A, 261, 579 Gathier, R., \& Pottasch, S. R. 1988, A\&A, 197, 266

Gathier, R., Pottasch, S. R., \& Pel, J. W. 1986, A\&A, 157, 171 Grevesse, N., Asplund, M., \& Sauval, A. J. 2007, Sp. Sci. Rev., 130, 105 Gregory, P. C., Vavasour, J. D., Scott, W. K., et al. 1994, ApJS, 90, 173 Guiles, S., Bernard-Salas, J., Pottasch, S. R., et al. 2007, ApJ, 660, 1282 
Henry, R. B. C., Kwitter, K. B., \& Balick, B. 2005, AJ, 127, 2284

Higdon, S. J. U., Devost, D., Higdon, J. L., et al. 2004, PASP, 116, 975

Houck, J. R., Appleton, P. N., Armus, L., et al. 2004, ApJS, 154, 18

Hummer, D. G., \& Storey, P. J. 1987, MNRAS, 224, 801

Karakas, A. I. 2003, Thesis, Monash Univ. Melbourne (see also Karakas, A., \& Lattanzio, J. C. 2007, PASA 24, 103)

Kerber, F., Mignani, R. P., Guglielmetti, F., \& Wicenec, A. 2003, A\&A, 408, 1029

Kingsburgh, R. L., \& Barlow, M. J. 1994, MNRAS, 271, 257

Milingo, J. B., Kwitter, K. B., Henry, R. B. C., et al. 2002a, ApJS, 138, 279

Milingo, J. B., Henry, R. B. C., \& Kwitter, K. B. 2002b, ApJS, 138, 285

Porter, R. L., Bauman, R. P., Ferland, G. J., et al. 2005, ApJ, 622, L73

Pottasch, S. R., \& Acker, A. 1989, A\&A, 221, 123
Pottasch, S. R., \& Beintema, D. A. 1999, A\&A, 347, 974

Pottasch, S. R., Wesselius, P. R., Wu, C. C., et al. 1977, A\&A, 54, 435

Pottasch, S. R., Dennefeld, M., \& Mo, J.-E. 1986a, A\&A, 155, 397

Pottasch, S. R., Preite-Martinez, A., Olnon, F. M., et al. 1986b, A\&A, 161, 363

Pottasch, S. R., Beintema, D. A., \& Feibelman, W. A. 2000, A\&A, 363, 767

Pottasch, S. R., Beintema, D. A., Bernard Salas, J., \& Feibelman, W. A. 2001, A\&A, 380, 684

Pottasch, S. R., Beintema, D. A., Bernard Salas, J., et al. 2002, A\&A, 393, 285

Preite-Martinez, A., \& Pottasch, S. R. 1983 A\&A, 126, 31

Rauch, T. 2003, A\&A, 403, 709

Surendiranath, R., Pottasch, S. R., \& García-Lario, P. 2004, A\&A, 421, 1051

Torres-Peimbert, S., \& Peimbert. M. 1977, RMxAA, 2, 181

Wright, A. E., Griffith, M. R., Burke, B. F., \& Ekers, R. D. 1994, ApJS, 91, 111 